\section{Bildung für nachhaltige Entwicklung: Themen und Herausforderungen}

\section{G. de Haan, G. Kamp, A. Lerch, L. Mar- tignon, G. Müller-Christ, H. Nutzinger: Nachhaltigkeit und Gerechtigkeit. Grundlagen und schulpraktische Konse- quenzen. Berlin: Springer Verlag, 2008, 260 S., ISBN 978-3-540-85491-3, € 74,95 \\ Rezension von Jürgen Kopfmüller, ITAS}

Die wachsende Vielfalt und Komplexität von gesellschaftlichen und natürlichen Entwicklungsprozessen erfordern zugleich rasches wie auch hinreichend fundiertes politisches und gesellschaftliches Handeln. Wissen und Bildung in ausreichendem Umfang und in angemessener Qualität für Bürger und Entscheidungsträger stellen eine zentrale Voraussetzung für die Realisierung einer weltweit nachhaltigen Entwicklung dar. In dem hier vorgestellten Band werden die Planungs- und Handlungserfordernisse zur Umsetzung der Nachhaltigkeitsidee sowie die Möglichkeiten und Herausforderungen der Integration dieses Themenfelds in die Schulpraxis vorgestellt und diskutiert.

\section{Hintergrund}

Nach nunmehr über zwei Jahrzehnten intensiver wissenschaftlicher, politischer und gesellschaftlicher Debatten über das Leitbild der nachhaltigen Entwicklung sind viele Fragen hinsichtlich seiner ethischen Prägung, seiner theoretischen Fundierung, seiner konzeptuellen Ausgestaltung und seiner Umsetzung in unterschiedlichsten gesellschaftlichen Bereichen gestellt und von verschiedener Seite darauf Antworten gegeben worden. Weitgehende Einigkeit besteht mittlerweile darin, dass es im Kern zugleich um die Erhaltung der natürlichen Lebensgrundlagen und die Gewährleistung eines menschenwürdigen Lebens für alle Menschen gehen muss. Dabei kommt dem Postulat der gerechten Verteilung von Ressourcen, Chancen, Risiken, Belastungen etc. sowohl zwischen gegenwärtigen und künftigen als auch innerhalb der gegenwärtigen Generationen zentrale Bedeutung zu. Gleichwohl sind vielfach noch erhebliche Wissenslücken wie auch Kontroversen über die Frage des „richtigen Wegs“ und daraus resultierende Handlungsbedarfe zu konstatieren. Eine Vielzahl drängender und schwerwiegender Probleme verlangt jedoch nach einer möglichst raschen Umsetzung von mitunter sehr weit reichenden Maßnahmen.

Erforderlich ist also zum einen die Erarbeitung verbesserter Wissensgrundlagen für Entscheidungsträger, zum anderen die Fähigkeit wie auch die Bereitschaft der Handelnden, dieses Wissen in praktisches Handeln umzusetzen. Bildung und Ausbildung in ihren verschiedenen Facetten, aber auch der Bewusstseinsbildung kommen hierbei erhebliche Bedeutung zu. Bereits in der 1992 verfassten Agenda 21 wurde dem in einem eigenen Kapitel Rechnung getragen. Mit der im Jahr 2002 durch die UN-Vollversammlung beschlossenen Welt-Dekade „Bildung für nachhaltige Entwicklung" wird diese Thematik für die Jahre 2005 bis 2014 auf supranationaler Ebene verankert und institutionalisiert. Hiermit verbunden sind eine Vielzahl von Aktivitäten auf nationaler wie internationaler Ebene zur Integration entsprechender Inhalte in schulische Programme und Lehrpläne.

Das vorliegende Buch gliedert sich in drei Teile: Im ersten Teil werden die Charakteristika und Herausforderungen der Klimathematik und der sich daraus ergebende Handlungsbedarf skizziert. Der zweite Teil befasst sich mit den begrifflichen, philosophischen und normativen Grundlagen des Nachhaltigkeitsbegriffs, seiner Ausprägung über das Gerechtigkeitskonzept sowie den daraus abgeleiteten Planungs- und Handlungserfordernissen. Im dritten Teil steht die schulpraktische Umsetzung der präsentierten Grundideen in konkrete Lehr-/Lernziele und -inhalte im Mittelpunkt. Abschließend werden einige Empfehlungen für eine angemessene Umsetzung der erziehungswissenschaftlichen und -praktischen Gestaltungsaufgabe präsentiert.

\section{Herausforderungen und Handlungsbedarf}

Die Frage, weswegen Bildung für nachhaltige Entwicklung ein zentrales Thema darstellt und welche Erfordernisse und Herausforderungen damit verbunden sind, veranschaulichen die Autoren anhand des Themas Klimawandel. Dieses, 
in den letzten Jahren in zahlreichen Perspektiven bearbeitete Thema, ist für diesen Zweck in besonderer Weise geeignet, da hier die eng mit dem Nachhaltigkeitsleitbild verbundenen Aspekte der Langfristorientierung und der Langzeitverantwortung unmittelbar zu Tage treten.

Im ersten Teil des Buches werden auf nur wenigen Seiten die wesentlichen Charakteristika der Klimathematik und die wichtigsten Kontroversen der letzten Jahre in sehr verständlicher Weise skizziert. Hinweise auf die Komplexität der naturwissenschaftlichen Zusammenhänge, der Phänomene des Wandels, ihrer Folgen und deren Bewertung sowie auf die bestehenden Wissensunsicherheiten und -begrenztheiten fehlen hier ebenso wenig wie die auf die verschiedenen - durch begrenztes Wissen aber auch durch unterschiedliche Interessenlagen bedingten - Kontroversen.

Kritisch anzumerken ist, dass die neben der Langzeitperspektive ebenso zentrale globale Dimension des Klimawandels über die Andeutung der naturwissenschaftlichen Zusammenhänge hinaus von den Autoren nicht thematisiert wird. Dies gilt v. a. für die politische Perspektive, die etwa in der Frage der Verteilung von Verantwortung sowohl für die Ursachen des Klimawandels als auch für den Umgang mit den negativen Wirkungen zum Ausdruck kommt. Es sind gerade die damit zusammenhängenden Verteilungs- und Gerechtigkeitsfragen, an denen sich die Debatten und Kontroversen festmachen und deren gemeinschaftliche Behandlung eine wesentliche Voraussetzung für tragfähige Handlungsstrategien darstellt.

Angesichts der Komplexitäten des Problemfelds Klimawandel und der bestehenden Unsicherheiten und Begrenztheiten des Wissens mahnen die Autoren zu Recht eine besondere „Urteilskraft" der Menschen an. Sie verstehen diese - in Anlehnung an Immanuel Kant - als die Fähigkeit des Einzelnen, selbständig und im Diskurs mit anderen sowie unter Verwendung nicht nur ökonomischer sondern auch ethischer Maßstäbe Bewertungen angemessen vornehmen und darauf basierend Entscheidungen treffen zu können.

\section{Nachhaltigkeitsbegriff und Gerechtigkeitskonzept}

Im zweiten Teil skizzieren die Autoren die Leitbilder Nachhaltigkeit und Gerechtigkeit sowie die sich daraus ergebenden Handlungserfordernisse. Es wird - zu Recht - darauf hingewiesen, dass über das nicht selten, in Anlehnung an Kant, kommunizierte Verständnis des Nachhaltigkeitsleitbilds als „regulative Idee“ hinaus begriffliche Klärungen und kriterielle Präzisierungen notwendig sind, wenn daraus praktikable Anleitungen für gemeinsames Handeln entstehen sollen. Bei den forstwirtschaftlichen Ursprüngen des 18. und 19. Jahrhunderts beginnend rekonstruieren die Autoren Nachhaltigkeit als Lebensvorsorge, d. h. als längerfristige Sicherung der Lebensgrundlagen, basierend auf regenerativen und nichtregenerativen Ressourcen. Am Beispiel des Umgangs mit der Verknappung nicht-regenerativer Ressourcen wird auf die Komplexität, Globalität, unterschiedliche Interessenslagen und zugleich bestehende Kooperationserfordernisse hingewiesen, was entsprechend veränderte Planungs- und Handlungserfordernisse mit sich bringt.

Die Autoren unterscheiden dabei zwischen einem sog. „planungsrationalen“ und einem „gerechtigkeitssensitiven“ Nachhaltigkeitsverständnis. Ersteres definieren sie als eine zweckmittel-abgestimmte Langfristplanung, die auf die ökologischen und ökonomischen Aspekte der globalen Ressourcenverknappung fokussiert ist. Entscheidend ist hier, dass zum einen die Sicherung der langfristigen Handlungsmöglichkeiten eines Systems im Mittelpunkt steht, zum anderen hierfür alleine die gegenwartsbezogene (intragenerative) Sicht („Jetzt-für-jetzt-Präferenzen") und die zukunftsbezogene (intergenerative) Sicht („Jetzt-für-dann-Präferenzen“) des oder der Planenden zu einem Ausgleich gebracht werden sollen. Die Handlungserfordernisse hierfür bestimmen sich alleine aus der zu treffenden Wahl der bestgeeigneten Mittel zur Erreichung der gesetzten Ziele bzw. Zwecke.

Dem stellen die Autoren ein gerechtigkeitssensitives Nachhaltigkeitsverständnis gegenüber, basierend auf den Grundideen des BrundtlandBerichts. Grundrationalität ist hier, von konfligierenden bzw. unvereinbaren Zwecksetzungen der Handelnden auszugehen, zwischen denen ein gerechter Ausgleich hergestellt werden soll.

Zur Rechtfertigung von Handlungsplänen, die den Verzicht auf eigene Zweckerreichung zu Gunsten anderer bzw. künftiger Akteure beinhal- 
ten, stellen die Autoren - in partieller Anlehnung an Kant - den Begriff der Moral in den Mittelpunkt. Sie wird verstanden als soziale Ressource zur Kontrolle von Macht zur Durchsetzung individueller Interessen Bildung ist dabei als eine wesentliche Ressource anzusehen, um Interessensabwägungen angemessen vornehmen und Strategien zur Minderung von Verknappungsproblemen entwickeln zu können.

\section{Nachhaltigkeit in Lehr- und Lernprozessen}

Der dritte Teil des Buches beschäftigt sich mit der Frage der schulpraktischen Umsetzung einer Bildung für nachhaltige und gerechte Entwicklung in Deutschland vor dem Hintergrund der Überlegungen der beiden vorherigen Kapitel. Besonderes Gewicht wird dabei auf eine Integration der Aspekte v. a. intergenerativer Gerechtigkeit in das bisherige Konzept des Kompetenzerwerbs im Rahmen der laufenden Aktivitäten zur „Bildung für nachhaltige Entwicklung“ gelegt. Zunächst wird der normative Rahmen hierfür entlang der Begriffe Selbstzwecklichkeit, Überwältigung- bzw. Indoktrinationsverbot sowie Kontroversitätsgebot beschrieben. Eine verantwortliche Schule soll nachhaltigkeitsrelevante Werte nicht appellativ vermitteln, sondern Kompetenzen, um sich selbst eine Meinung bilden, Kontroversen reflektieren und Werte umsetzen zu können. Pädagogik kommt damit eher die Rolle der „Eröffnung von Möglichkeiten“ $z u$.

Im Anschluss daran werden vier Themenfelder in je einem Kapitel näher beleuchtet: (a) wesentliche der Umsetzung nachhaltiger Entwicklung entgegenstehende Faktoren, (b) die zu vermittelnden Kompetenzen, (c) die Themen ökonomische Kompetenz und nachhaltiger Konsum sowie (d) schulpraktische Überlegungen zum Kompetenzkonzept und zu Lernkonzeptionen für seine Umsetzung.

$\mathrm{Zu}$ a) Damit Schüler nicht nur die richtigen (nachhaltigen) Zwecke setzen, sondern auch entsprechende Wirkungen entfalten können, sollten ihnen neben Sachinformationen auch Kompetenzen vermittelt werden, um bestehende individuelle wie gesellschaftliche Umsetzungshemmnisse erkennen und überwinden zu können.
Ebenso unerlässlich sind kognitive Fähigkeiten zum Umgang mit unvollständigem, unsicherem (Wahrscheinlichkeiten) oder überkomplexem Wissen (Komplexitätsreduktion), mit der häufig notwendigen längerfristigen Perspektive sowie mit risikobehafteten Entscheidungssituationen.

$\mathrm{Zu} \mathrm{b)} \mathrm{Hier} \mathrm{erläutern} \mathrm{die} \mathrm{Autoren} \mathrm{zunächst}$ das Konzept des Erwerbs von Gestaltungskompetenzen als Kern einer Bildung für nachhaltige Entwicklung, basierend auf dem Konzept der Schlüsselkompetenzen der OECD. Dort werden die drei Kompetenzkategorien „Interaktive Verwendung von Medien und Tools“, „Interagieren in heterogenen Gruppen“ und „Eigenständiges Handeln" unterschieden und in Teilkompetenzen gegliedert. Hierzu schlagen die Autoren die Ergänzung dreier weiterer Teilkompetenzen vor, basierend auf den Überlegungen des vorherigen Kapitels, um eine angemessenere Vermittlung und Reflektion der Gerechtigkeitsthematik und damit zusammenhängender Fragen des Umgangs mit Unsicherheiten oder Risiken in Entscheidungssituationen zu ermöglichen.

$\mathrm{Zu} \mathrm{c)}$ Als Beispiel für einen Bereich, in dem diese Kompetenzen täglich angewendet werden (können), betrachten die Autoren dann das Konsumverhalten von Jugendlichen und die Rolle, die ökonomische Kompetenz und ökonomische Entscheidungsrationalitäten sowie Medien und andere externe Einflüsse dabei spielen.

$\mathrm{Zu} \mathrm{d)} \mathrm{Ausgehend} \mathrm{von} \mathrm{der} \mathrm{Einschätzung,}$ dass dem Konzept „Bildung für nachhaltige Entwicklung" in der vergangenen Dekade durchaus eine Erfolgsgeschichte attestiert werden kann, versuchen die Autoren zum Abschluss eine Beurteilung des Kompetenzkonzepts. Seine stärker lebensweltliche Orientierung (und damit bessere Alltagstauglichkeit) und seinen Fokus auf Kontext- und Problem(lösungs)bezug anstatt spezifischem Fachwissen führen sie als Vorzüge an. V. a. organisatorisch und konzeptionell problematisch schätzen sie die Möglichkeiten eines Aufbaus vergleich- und messbarer Kompetenzkataloge und -standards ein.

Zum Abschluss formulieren die Autoren einige Handlungsempfehlungen, die sich an Entscheidungsträger und „Praktiker vor Ort“ richten. Sie fassen die im Buch behandelten Punkte zusammen und fokussieren auf die bei- 
den Aspekte, die die Autoren als bislang in der Debatte und Praxis einer Bildung für nachhaltige Entwicklung als besonders defizitär ansehen: die Reflektion von Konzepten der (generationenübergreifenden) Gerechtigkeit sowie die ökonomische Seite der Nachhaltigkeit.

\section{Fazit}

Mit dem vorliegende Band wollen die Autoren die Ergebnisse ihrer interdisziplinären Studie in den notwendigen Diskurs darüber einbringen, wie geeignete schulische Angebote zur Bewältigung von Planungs- und Handlungsentscheidungen für mehr Nachhaltigkeit aussehen und wie entsprechende methodische Grundlagen und organisatorische Voraussetzungen für deren Realisierung beschaffen sein sollten. Sie wenden sich damit zunächst an Leser aus der (hoch)schulischen Praxis und aus der Wissenschaft, sprechen aber auch an der Thematik Interessierte an. Sie geben dem Leser einen recht umfassenden Einblick in die Nachhaltigkeits- und die Gerechtigkeitsthematik und liefern (schul)politischen Entscheidungsträgern Informationen und Argumente, welche Kompetenzen Schülern vermittelt werden sollten, damit sie diese Themen angemessen reflektieren und umsetzen können. Angesichts des nach wie vor unbefriedigenden Situation der Integration des Nachhaltigkeitsthemas in den Schulalltag stellt das Buch damit einen wichtigen Beitrag zur Überwindung bestehender Defizite dar. Vor diesem Hintergrund sind die folgenden kritischen Anmerkungen einzuordnen.

Die rund 230 Seiten sind überwiegend auf einem der Komplexität der Thematik angepassten und wissenschaftlich hohen Niveau geschrieben. Die Verwendung von erläuternden Beispielen zu den theoretischen Ausführungen erleichtert das Verständnis. Das macht das Buch insgesamt sehr gehaltvoll. Allerdings sind die teilweise in die Tiefe gehenden systemtheoretischen Passagen mitunter auch für mit der Thematik Vertraute schwierig und ermüdend zu lesen und erschweren das Weiterdenken in Richtung der später angesprochenen schulpraktischen Konsequenzen. Es wäre hilfreich gewesen, im Sinne des Untertitels des Buches die Beschreibung der Grundlagen der Thematik mit noch mehr, konkreteren und entlang eines spezi- ellen Themenfelds gewählten Beispielen zu untermauern, die dem Leser einen leichteren Bezug zur praktischen Ebene ermöglichen.

Der für die Verdeutlichung von Handlungsrationalitäten bei Entscheidungen über Ressourcennutzungen vorgenommenen Trennung zwischen den Prinzipien der auf die Sicherung von Handlungsmöglichkeiten fokussierten ,planungsrationalen Nachhaltigkeit" und der ,gerechtigkeitssensitiven Nachhaltigkeit" kommt in dem Buch eine erhebliche Rolle zu. Sie wird immer wieder im Text aufgegriffen. Begründet wird dies v. a. mit dem sehr theoretischen Argument, dass nur voneinander unabhängig konzipierte Prinzipien teilweise oder gar nicht miteinander verträglich sein bzw. sich ergänzen können. Dieses Vorgehen mag aus quasi didaktischen Gründen gerechtfertigt sein, um „Idealtypen“ zu zeichnen und damit ,gute“ und „schlechte“ Verhaltensweisen voneinander abzugrenzen. Die hier vorgenommene Differenzierung zeichnet jedoch zu sehr schwarz-weiß und diskreditiert zumindest implizit den Planungsbegriff zu Unrecht. Den Nachhaltigkeitsdebatten der letzten 10 bis 15 Jahre folgend muss „,kluges“ (d. h. nachhaltiges) Handeln eine Mischung aus beiden Prinzipien darstellen: Die Sicherung von gesellschaftlicher Handlungsfähigkeit kann nicht ohne die Überzeugung der Menschen gelingen, dass es dabei gerecht zugeht; und die Realisierung von gerechtem Ausgleich ist ohne eine handlungs-(bzw. planungs)fähige Gesellschaft nicht möglich

Anzumerken ist noch, dass neben der ausführlichen Behandlung der Lehrinhalte die Frage nach den hierfür erforderlichen Lehrformen etwas zu kurz kommt. Sie beschränkt sich im Wesentlichen auf den Hinweis, dass der Projektunterricht das geeignete Mittel zur angemessenen Vermittlung von Bildung für nachhaltige Entwicklung darstellt. Hier wären etwas konkretere Hinweise auf mögliche Unterrichtseinheiten und -gestaltungsformen interessant und hilfreich gewesen, gerade auch mit Blick auf den Adressatenkreis.

Abschließend sei noch einmal herausgestellt, dass es sich hier um eine der wenigen, und schon deswegen wichtigen Publikationen handelt, die einem breiteren Leserkreis das Thema Schulbildung für nachhaltige Entwicklung inhaltlich und anwendungsbezogen nahe zu bringen versuchen. 
Umso sympathischer wirkt daher der - zwar durchaus angemessene, allerdings heutzutage nicht selbstverständliche - Hinweis der Autoren, dass das Thema nicht neu zu erfinden ist, sondern es darum geht, bereits Vorhandenes zu prüfen und gegebenenfalls daran anzuknüpfen. In diesem Sinne sei auf eine Arbeit von Emmrich und Melzer hingewiesen. Darin wurde über die Möglichkeiten und erste Erfahrungen mit der Verwendung des in der Helmholtz-Gemeinschaft entwickelten integrativen Nachhaltigkeitskonzepts ${ }^{1}$ als Bestandteil des Unterrichts in der Sekundarstufe II berichtet. ${ }^{2}$ Dieses Konzept ist im Kern an der Gerechtigkeitsidee ausgerichtet und nach wie vor eines der wenigen, das theoretisch-konzeptionell ausgereift und zugleich so weit inhaltlich konkretisiert ist, dass es prinzipiell als eine Basis für den Schulunterricht dienen kann. Es waren v. a. die Komplexität des Konzepts und eine für die Altersstufe angemessene sprachliche Verarbeitung, wo die Autoren damals aus ihrer eigenen Arbeit und den Rückmeldungen aus den Schulen primär Überarbeitungs- und Weiterentwicklungsbedarf gesehen hatten. Hier hätte beispielsweise eine Möglichkeit bestanden, basierend auf vorhandenen Erfahrungen noch konkretere Umsetzungsschritte in Richtung angemessener Lehrinhalte und -formen zu diskutieren und vorzuschlagen, als es die Autoren in diesem Band getan haben. Mindestens ergibt sich jetzt, quasi am Ende dieses Buches, die Perspektive, auf diesem Weg einen Schritt weiter zu gehen. Es wäre immens wichtig, um die bestehenden Defizite im Schulunterricht abzubauen, und daher aller Mühen wert.

\section{Anmerkungen}

1) Siehe Kopfmüller, J.; Brandl, V.; Jörissen, J. et al., 2001: Nachhaltige Entwicklung integrativ betrachtet. Konstitutive Elemente, Regeln, Indikatoren. Berlin

2) Emmrich, R.; Melzer, M., 2006: Das integrative Nachhaltigkeitskonzept der HGF als Baustein der Bildung für eine nachhaltige Entwicklung. In: Kopfmüller, J. (Hg.): Ein Konzept auf dem Prüfstand. Das integrative Nachhaltigkeitskonzept in der Forschungspraxis. Berlin

\section{$\langle\langle\rangle$}

\section{Elektronische Petitionen \\ Potenziale zur Modernisierung und Stärkung von Bürgerbeteiligung}

\section{U. Riehm, Chr. Coenen, R. Linder, C. Blümel: Bürgerbeteiligung durch E-Petitionen - Ana- Iysen von Kontinuität und Wandel im Petiti- onswesen. Berlin: edition sigma, 2009, 278 S., ISBN 978-3-8360-8129-0, $€$ 24,90}

\section{Rezension von Tobias Escher, Oxford Internet Institute}

Das Medium Internet ist aus der politischen Kommunikation nicht mehr wegzudenken. Gleichzeitig ist seine Bedeutung für den politischen Prozess und seine Wirkung auf die an diesen Prozessen beteiligten Akteure nach wie vor umstritten. Der Deutsche Bundestag hat sich den Hoffnungen von erhöhter Transparenz, Legitimität und Beteiligung durch den Einsatz neuer Medien angeschlossen und im Juni 2005 einen Modellversuch gestartet, der sowohl die Einreichung als auch die Mitzeichnung von Petitionen über das Internet ermöglicht. Das Büro für Technikfolgen-Abschätzung beim Deutschen Bundestag (TAB) hat diesen Modellversuch im Rahmen einer groß angelegten Studie von Oktober 2006 bis Mai 2007 evaluiert. Diese ist mittlerweile in Buchform bei edition sigma erschienen.

Ziel war es, die Erfahrungen von Petenten und Bundestag mit dieser neuen Form der Einreichung und Mitzeichnung von Petitionen in Erfahrung zu bringen und gleichzeitig festzustellen, inwieweit diese zu Veränderungen im deutschen Petitionswesen führen. Den Kern dieser Evaluation bildeten Umfragen und Interviews sowohl mit traditionellen und elektronischen Petenten als auch mit Abgeordneten des Petitionsausschusses sowie der Bundestagsverwaltung. Weiterhin wurde eine Analyse der Diskussionsforen durchgeführt und die genutzte Software einer eingehenden Prüfung unterzogen.

Dabei beschränken sich die Autoren keineswegs nur auf die Untersuchung des Modellversuchs, sondern nehmen sich vielmehr das Petitionswesen als Ganzes und die Rolle elektronischer Petitionen im Besonderen vor. Die Betrachtung erfolgt sowohl in Deutschland als auch im internationalen Vergleich, wofür u. a. Studien 